\title{
An Adaptive Threshold Method for Spectrum Sensing in Multi-Channel Cognitive Radio Networks
}

\author{
Ali Gorcin *, Khalid A. Qaraqe*, Hasari Celebi *, Huseyin Arslan ${ }^{\dagger}$ \\ *Dept. of Electrical and Computer Engineering, Texas A\&M University at Qatar, PO Box 23874, Education City, Doha, Qatar \\ Emails: ali.gorcin, khalid.qaraqe, hasari.celebi@qatar.tamu.edu \\ ${ }^{\dagger}$ Department of Electrical Engineering, University of South Florida, 4202 E. Fowler Ave., ENB-118, Tampa, FL, 33620, USA \\ Email: arslan@eng.usf.edu
}

\begin{abstract}
Opportunistic spectrum access feature of cognitive radio systems is a method to improve frequency underutilization of wireless spectrum. One of the techniques for detecting the unused bands is the energy detection for which selection of the threshold defines detection performance. In this paper, the adaptive threshold method is proposed as an alternative approach to estimate the threshold as a function of first and second order statistics of recorded signals. The proposed method does not require estimation of noise variance or signal to noise ratio and aims to minimize the effects of impairments introduced by wireless channel and non-stationary noise. The simulation results indicate that adaptive threshold has low false alarm and missed detection rates that can satisfy the detection requirements of multi-channel cognitive radios for either narrow or wideband spectrum sensing, when the standard deviation coefficient is selected properly.
\end{abstract}

\section{INTRODUCTION}

Wireless spectrum became a more scarce and valuable resource due to requirement of more bandwidth for next generation networks and because of increasing number of users. Even though the spectrum allocation charts indicate that there is not much space left in the frequency spectrum, in practice the spectrum is not efficiently allocated because the communications systems using the frequency spectrum dynamically such as cellular technologies, are assumed to be transmitting in a static fashion such as in the case of television transmitters. Federal Communications Commission's (FCC) Spectrum Policy Task Force report [1] investigates underutilization of the spectrum by precise definition of the spectral efficiency and makes specific recommendations for fostering uniform throughput. Moreover, FCC's Notice of Proposed Rule Making which is issued after the task force's report, recommends employment of flexible and reliable communications systems, such as cognitive radios for efficient spectrum use [2].

The spectrum sensing feature of cognitive radios (CRs) introduces techniques for detecting communications opportunities in wireless spectrum by the employment of secondary users with low-priority access to the spectrum. Three signal processing methods used for spectrum sensing are matched filter, cyclostationary feature extraction, and energy detection [3]. First two are coherent detection techniques with better detection probability than non-coherent energy detection [4]. However, the coherent detectors require a priori information.
Matched filter provides optimal detection by maximizing signal to noise ratio (SNR) but requires demodulation parameters. Cyclostationary feature detection can detect random signals depending on their cyclic features even if the signal is in the background of noise but it requires information about the cyclic characteristics. Energy detection technique is applied by setting a threshold for detecting the existence of the signal in the spectrum. Even though it is simpler than the match filtering and cyclostationary feature detection, it requires at least $O\left(1 / S N R^{2}\right)$ samples for detection [5] and it has fundamental problems: (i) susceptibility to changing noise levels, (ii) can not distinguish modulated signals, noise and interference. Therefore it can not treat primary users, secondary users and noise in different ways, (iii) can not detect the direct sequence spread spectrum signals with very low SNR [6].

Despite the aforementioned drawbacks, energy detection can provide lower sensing period than other methods and it is applicable to multiple channels for wideband sensing. Hence energy detection is the most studied technique among all sensing methods. Research to improve performance of the energy detectors includes cooperative sensing with multiple CRs [7] and multi-band joint detection which evaluate each frequency band separately [8]. Various double threshold methods [9], [10], [11] are also proposed to improve the threshold selection process. Besides, an adaptive threshold setting algorithm is introduced for single channel duty cycle estimation [12]. On the other hand, [13] defined a system level threshold adaptation algorithm taking the detection performances of the thresholds selected for previous frequency sweeps into consideration.

Even though the detection performance can be improved by the methods mentioned above, threshold selection is the most important process that defines the performance of the energy detectors. Therefore, in this paper, it is aimed to investigate how can the threshold selection process be optimized with the knowledge of the minimum number of parameters that contribute to the threshold estimation. An adaptive threshold algorithm depending on the first and second order statistics of the recorded spectrum is proposed and detection performance of the adaptive threshold method is investigated. The system model for the signals that the proposed method will be applied to is presented in Section II. Performance metrics and the theoretical analysis of adaptive threshold constitute Section 


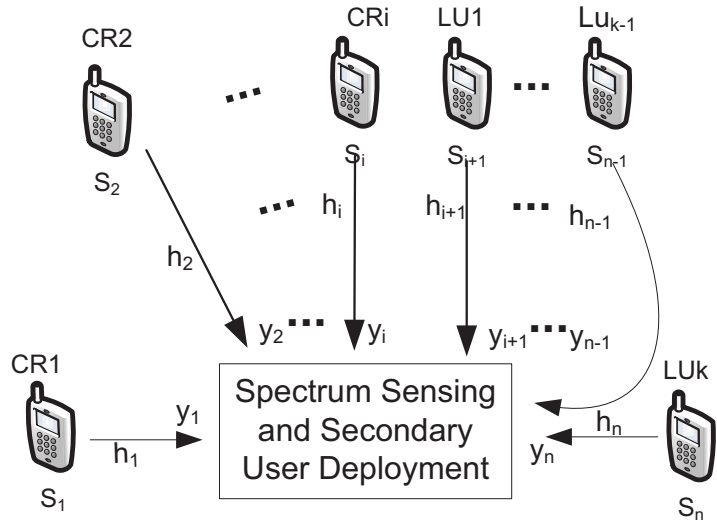

Fig. 1. System Model for Spectrum Sensing

III. Section IV details the numerical results and Section V provides the conclusion.

\section{System Model}

It is assumed that cognitive radio is sensing multiple channels or a chunk of the frequency spectrum that may be occupied by multiple signals. Therefore, transmit signal model can be given by

$$
x(t)=A s(t),
$$

where $s(t)=\left[s_{1}(t), \ldots, s_{n}(t)\right]^{T}$ represents $n$ independent signals and $T$ denotes transposition. $A$ is $n \times n$ coefficient matrix with $a_{i, j}$ elements where $i, j=1, \ldots n$ and $x(t)$ represents $n$ transmit signals where $x_{j}(t)=\sum_{i=1}^{n} a_{i, j} s_{j}(t)$. The proposed system model is illustrated in Fig. 1 and after digital conversion and modulation, each independent signal passes through a different channel as indicated in the figure. The channel for $x_{j}(t)$ can be modeled as a time-variant linear filter

$$
h_{j}(t)=\sum_{i=1}^{L_{j}} h_{i}(t) \delta\left(t-\tau_{i}\right),
$$

where $L_{j}$ is the number of taps for the channel $h_{j}$ and $\tau_{i}$ is delay for each tap. It is assumed that the taps are sample spaced and the channel is constant for a symbol but timevarying across multiple symbols. Each of the emitters in the spectrum can be a legacy user (primary) or a secondary user.

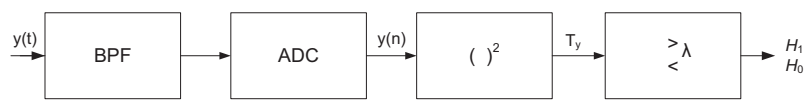

Fig. 2. Block Diagram of Energy Detector

Block diagram of proposed the energy detection scheme is given in Fig. 2. The transmitted signal is received along with noise at the receiver. Therefore, baseband model of a single received signal at the input of the energy detector, after down conversion can be given as

$$
\begin{aligned}
y_{j}(t) & =e^{2 \pi j \xi t}\left[x_{j}(t) \star h_{j}(t)\right]+w(t) \\
& =e^{2 \pi j \xi t} \int x_{j}(\tau) h_{j}(t-\tau) d \tau+w(t),
\end{aligned}
$$

where $\star$ is the convolution operation and the $j$ at the exponential term is the imaginary unit. When the channel model given in (2) is considered received signal becomes

$$
y_{j}(t)=e^{2 \pi j \xi t} \sum_{i=1}^{L_{j}} x_{j}\left(t-\tau_{i}\right) h_{i}(t)+w(t),
$$

where $\xi$ is frequency offset due to inaccurate frequency synchronization and $w(t)$ corresponds to additive white Gaussian noise (AWGN) sample with zero mean and variance of $\sigma_{w}^{2}$. The composite received signal then becomes

$$
y(t)=\sum_{j=1}^{n} y_{j}(t) .
$$

The received signal is sampled with sampling time of $\Delta t$ at the analog to digital converter (ADC) and discrete-time received signal can be represented in vector notation by

$$
y(n)=[y(1), y(2), \ldots, y(N)] .
$$

where $n=1, \ldots, N$. After the received signal is digitized, discrete samples are squared and averaged to acquire received signal energy and the estimated energy is compared with the threshold $\lambda$ and the decision is made whether the signal is present or not as follows

$$
T_{y}=\frac{1}{N} \sum_{i=1}^{N}(y(i))^{2} \gtrless \lambda
$$

\section{Performance Analysis}

The decision problem for the energy detection scheme can be formulated in a binary hypothesis form

$$
\begin{aligned}
& H_{0}: y(n)=w(n) \quad(\text { no signal present }) \\
& H_{1}: y(n)=h(n) \times s(n)+w(n) \quad(\text { signal present })
\end{aligned}
$$

$s(n)$ represents a transmitted signal in the given frequency band and assumed to be independent and identically distributed (iid) with zero mean and $\sigma_{s}^{2}$ variance. $w(n)$ is the additive white Gaussian noise (AWGN) and assumed to be iid with variance of $\sigma_{n}^{2} . h(n)$ is the temporary amplitude gain of the channel. SNR can be defined in terms of the signal and noise variance as

$$
S N R=\sigma_{s}^{2} / \sigma_{n}^{2} .
$$

Energy detection theory [14] indicate that $T_{y}$ is a random variable with central and non-central chi-square distributed probability distribution function (PDF) under $H_{0}$ and $H_{1}$ respectively. When the observation interval $N$ is large enough, the PDF of $T_{y}$ can be approximated as Gaussian distribution

$$
\begin{aligned}
& \left.f\left(T_{y}\right) \backsim \mathcal{N}\left(\sigma_{n}^{2}, 2 \sigma_{n}^{2} / N\right) \quad \text { (under } H_{0}\right) \\
& f\left(T_{y}\right) \sim \mathcal{N}\left(\sigma_{y}^{2}, 2 \sigma_{y}^{2} / N\right) \quad\left(\text { under } H_{1}\right)
\end{aligned}
$$

where $\sigma_{y}^{2}=\sigma_{s}^{2}+\sigma_{n}^{2}$. When the derivations in [15] are taken into consideration probability of signal detection can be given by

$$
P_{\text {det }}=Q\left(\sqrt{N / 2}\left(\frac{\lambda}{\sigma_{n}^{2}(1+S N R)}-1\right)\right)
$$

and the probability of false alarm can be written as

$$
P_{f a}=Q\left(\frac{\lambda-\sigma_{n}^{2}}{\sigma_{n}^{2} / \sqrt{N / 2}}\right)
$$


Under $H_{1}$ hypothesis, for a fixed target of detection probability the threshold is derived as [15]

$$
\lambda_{d}=\sigma_{n}^{2}(1+S N R)\left(1+\frac{Q^{-1}\left(p_{d}\right)}{\sqrt{N / 2}}\right),
$$

and for a constant false alarm rate $\left(P_{f a}\right)$ the threshold is given by

$$
\lambda_{f a}=\sigma_{n}^{2}\left(1+\frac{Q^{-1}\left(p_{f a}\right)}{\sqrt{N / 2}}\right) .
$$

(14) and (15) indicate that the threshold estimation is dependent of noise variance, SNR, number of the samples, and required detection or false alarm probability. Therefore, in the literature, noise variance and SNR are either assumed to be known or methods to estimate these parameters are proposed. For instance, [16] proposes a combined approach which estimate the noise floor via histogram first, and then applies a constant false alarm rate (CFAR) detector. Performance of the thresholds are investigated when an estimate of noise variance from side or empty bands is used instead of exact values in [15]. Besides, in general it is also assumed that the noise floor is stationary. However, in practice, this assumption may become invalid due to the wireless channel and other impairments especially when the multi-band detection is considered. [10] and [13] introduce the channel impairments in the hypothesis model but these effects are assumed to be either deterministic or non-fading. On the other hand, in the literature, it is aimed to come up with a single threshold value. However, especially for the multi-band or wide-band detection single threshold value may reduce the detection performance.

In this study, we adopted the adaptive threshold method from the literature of image processing to overcome the limitations mentioned above. The adaptive threshold method defines the threshold as linear function of recorded signal's mean and standard deviation. We will consider and discuss two types of adaptive thresholds: first, the adaptive threshold which fluctuate with the signal and second, the computationally optimal version which is estimated as a single value for the all spectrum in once.

In the context of the adaptive threshold, we slightly modify the energy detector proposed in Fig. 2. The initial bandpass filter stage is removed and after the analog to digital conversion N-point fast Fourier transform (FFT) is applied instead of taking the square and averaging the received signal. This approach brings more flexibility to wideband multi-channel signal processing and provides sensing of multiple signals simultaneously in a simple way [5]. The frequency domain representation of the received signal is acquired by applying FFT over $y(n)$ as

$$
Y(u)=\sum_{n=1}^{N} y(n) e^{-j 2 \pi u \frac{n}{N}}
$$

where $u=0, \ldots, N-1$. When the focus is on the parameters in hand, it's known that there are $N$ (FFT size) samples of sequence for spectral representation of the recording. Also when the practical cases considered, because of the dynamic range of recording hardware, energy bearing samples lower than the minimum value of the hardware's dynamic range will be represented at the dynamic range minima. These samples accumulated at the bottom of the spectrum will carry least of the information while the samples with highest power levels will carry most of the information about the wireless occupant signal. Therefore, it is plausible to divide the signal samples into two groups: One group starting from the bottom of the spectra should constitute the noise floor and the other group of samples should be evaluated as the information bearing samples for wireless signals. Therefore, selection of the threshold level for energy detection is extremely important and has its overlapping counterpart in the field of image processing: The adaptive or dynamic threshold method is employed to determine the level of image intensity boundary for converting the color or the gray scale images to the binary images, the process which is called image binarization [17]. According to this method, the image pixel with the intensity level lower than the calculated threshold is marked as being zero and the pixel with higher and equal level of intensity to the threshold is marked as being 1 during the binarization process. Starting from the initial pixel of the image, the level of the threshold calculated separately for each pixel taking the information gathered from previous pixels into consideration. If each pixel of a digital image, under the image field size defined with the window of $W(N, N)$ is represented by $I(u, v)$, the threshold to decide the intensity level for each pixel can adaptively be calculated by

$$
\lambda_{u, v}=\mu_{W}(u, v)+k \sigma_{W}(u, v) \quad \forall I(u, v),
$$

where

$$
\mu_{W}(u, v)=\frac{1}{u \times v} \sum_{p=1}^{u} \sum_{r=1}^{v} I(p, r)
$$

and

$$
\sigma_{W}(v, v)=\sqrt{\frac{1}{u \times v} \sum_{p=1}^{u} \sum_{r=1}^{v}\left(I(p, r)-\mu_{W}(u, v)\right)^{2}}
$$

where $k$ is the standard deviation coefficient which depends on the a priori information about the application. For instance, it is generally selected between zero and one in the digital image processing applications. (17) imply that estimation of the noise variance and SNR are not essential and the effect of fluctuations in the noise floor is minimized, however selection of the standard deviation coefficient, $k$, defines the detector performance.

The adaptive threshold finds its validation in the fact that the standard deviation indicates the dispersion level of the spectral data around a mean value. If the data is fluctuating much, the dispersion of the recorded signal will change faster and the threshold will also vary faster. If the fluctuations is less, then threshold will also change slowly. From this point of view, computation of the mean and the standard deviation at each point and adjusting the threshold accordingly will provide an advantage against changes in the signal. However, because of the fact that the mean and standard deviation is calculated cumulatively, the threshold can not trace sharp changes in the frequency spectrum fast enough. Hence, the missed detection performance of the threshold can be deteriorated. This problem can be minimized by the introduction of optimal version of 
adaptive threshold. But before that, there will be another critical assumption which will also effect the performance of the proposed method. In traditional image processing applications, the binarization is performed by scanning the entire two dimensional area of interest. However, when the spectrum of wireless signals are considered, a single power value is assigned to each frequency point in the spectrum as (16) implies and there is no need for vertical search. Hence, for the adaptive threshold, instead of two dimensional digital image of $I(u, v)$ the wireless signal can be represented with $Y(u)$ under the boundary of $W(N)$. Formulas can be modified and rewritten for the specific case of spectral detection as

$$
\lambda_{u}=\mu_{W}(u)+k \sigma_{W}(u) \quad \forall Y(u)
$$

and

$$
\begin{gathered}
\mu_{W}(u)=\frac{1}{u} \sum_{p=1}^{u} Y(p) \\
\sigma_{W}(u)=\sqrt{\frac{1}{u} \sum_{p=1}^{u}\left(Y(p)-\mu_{W}(u)\right)^{2}}
\end{gathered}
$$

The computationally optimal version of adaptive threshold can be obtained by calculating the mean and variance in once by

$$
\lambda_{N}=\mu_{Y}+k \sigma_{Y}
$$

where

$$
\mu_{Y}=\frac{1}{(N)^{2}} \sum_{p=1}^{N} Y(p)
$$

and

$$
\sigma_{Y}=\sqrt{\frac{1}{(N)^{2}} \sum_{p=1}^{N}\left(Y(p)-\mu_{Y}\right)^{2}} .
$$

\section{Simulation Results}

In this section, performance assessment of the proposed methods is discussed for simulated signals. A simulation setup is constructed with generating baseband signals which employ 16 QAM modulation with various symbol rates, each signal is consisting of 1024 symbols. Combination of root raised cosine and Gaussian filters are used for pulse shaping. The impacts of multipath channel is simulated in MATLAB by considering ITU-R M.1225 outdoor to indoor channel model which is compatible to the system model proposed in Section II.

(20) indicates the critical parameter which affects the performance of the proposed adaptive threshold: standard deviation coefficient. Therefore, in order to monitor the effect of selection of $k$ over detection performance we define the metrics that change the false alarm and missed detection rates when the standard deviation coefficient is fixed. One metric will be SNR because when the SNR of signals in the monitored spectrum change the distribution will also change. Second metric will be the ratio of spectral occupancy versus monitored multi-channel spectrum and it is defined by $B W_{o c c} / B W_{t o t}$, where $B W_{o c c}$ is the total occupied bandwidth over monitored spectrum $B W_{t o t}$. There can be one or more signals in the spectrum however all the signals are assumed to have same SNR value for the purpose of investigating the effects of spectral occupancy and also for the sake of simplicity.

$B W_{o c c} / B W_{\text {tot }}$ is set to the fixed value of 0.5 and the effect of changing SNR is monitored. Wireless signals are occupied on both $20 \mathrm{kHz}$ and $4 \mathrm{MHz}$ spectrum bands. False alarm and missed detection rates are calculated for each signal according to the corresponding SNR levels. A representative curve for decision statistics is obtained by averaging 50 statistics and results have been plotted in Fig.3 and Fig.4 for $k$ values varying between -2 and 4.4. Confidence levels for zero SNR value is also plotted in order to indicate the theoretical limit of the proposed approach. When practical SNR levels are of interest (e.g., SNR $>5 \mathrm{~dB}$ ), simulation results show that false alarm and missed detection rates have close numerical values for both $20 \mathrm{kHz}$ and $4 \mathrm{MHz}$ bands. This implies that, for the fixed rate of spectral occupancy, monitored bandwidth does not have significant effect on the selection of standard deviation coefficient.

Fig.5 depicts the general view of the effects of metrics over standard deviation coefficient. Optimum values of $k$ that minimize both false alarm and missed detection rates are marked for both adaptive (Fig.5 (a)) and optimum threshold (Fig.5 (b)). Note that $k$ should be increased as occupancy declines. The $k$ value resides in the range of 0 and 1 for adaptive threshold and varies from -1 to 0.5 for the optimal threshold. Please also note that optimum $k$ values increase with increasing SNR for adaptive threshold while they decrease with increasing SNR in the case of optimum threshold. Fig 6 illustrates the false alarm and missed detection rate comparison of adaptive and optimal threshold for 0.2 of $B W_{o c c} / B W_{t o t}$ and $15 \mathrm{~dB}$ of SNR. Optimal threshold has better detection rate because it does not have to trace the changes due to increasing and falling edges of the signals, however it has a wider search range for $k$ as indicated in Fig.5.

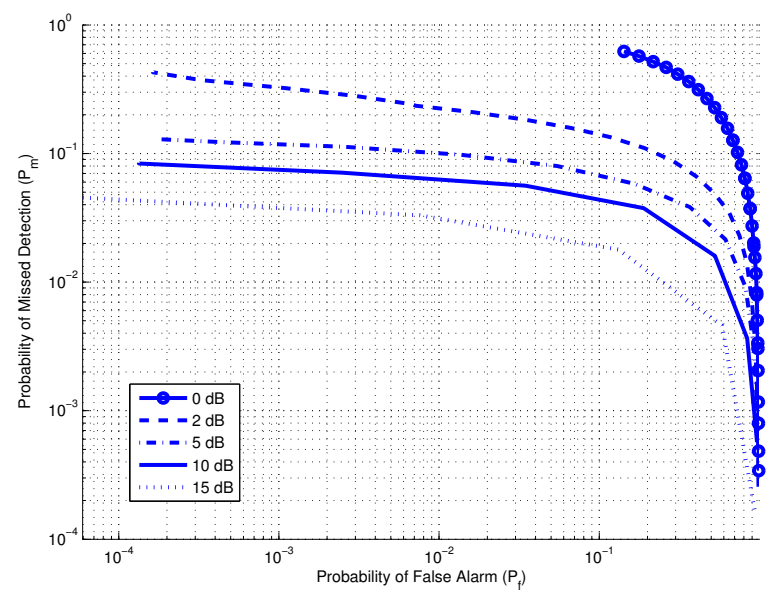

Fig. 3. Miss Detection/False Alarm Rate $\left(20 \mathrm{kHz}\right.$ Span, $\left.B W_{\text {occ }} / B W_{\text {tot }}=0.5\right)$

\section{Conclusion}

Estimation of energy threshold is the most important factor that affects the performance of the energy detection technique. There are many approaches for threshold selection however, in general, statistical procedures are dependent of the noise variance, SNR and the recording interval. In this paper, it is aimed to develop a threshold selection method based on the 


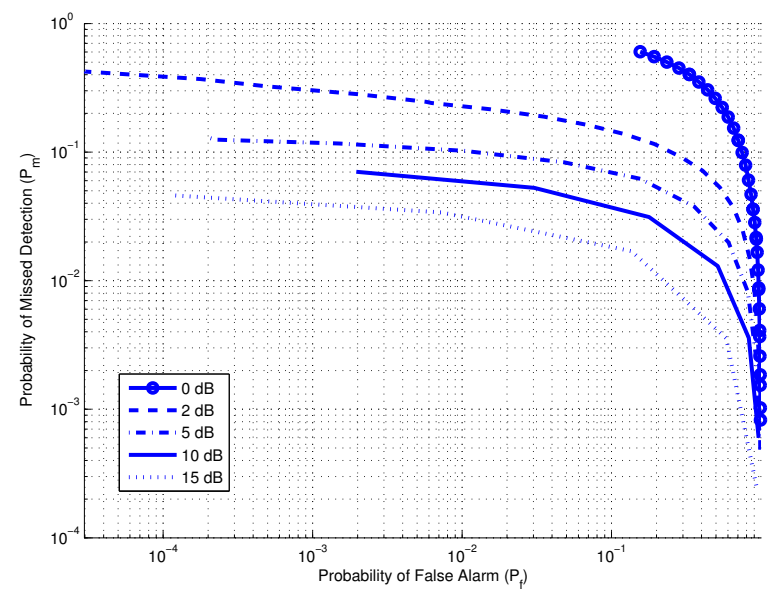

Fig. 4. Miss Detection/False Alarm Rate (4 MHz Span, $\left.B W_{o c c} / B W_{\text {tot }}=0.5\right)$
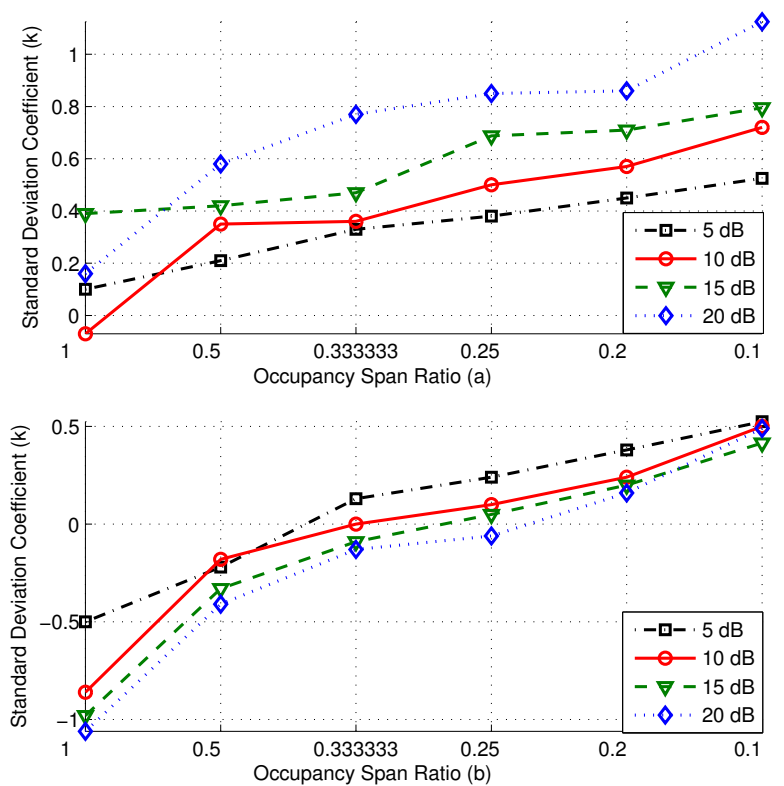

Fig. 5. Optimum $k$ values: SNR vs. Occupancy

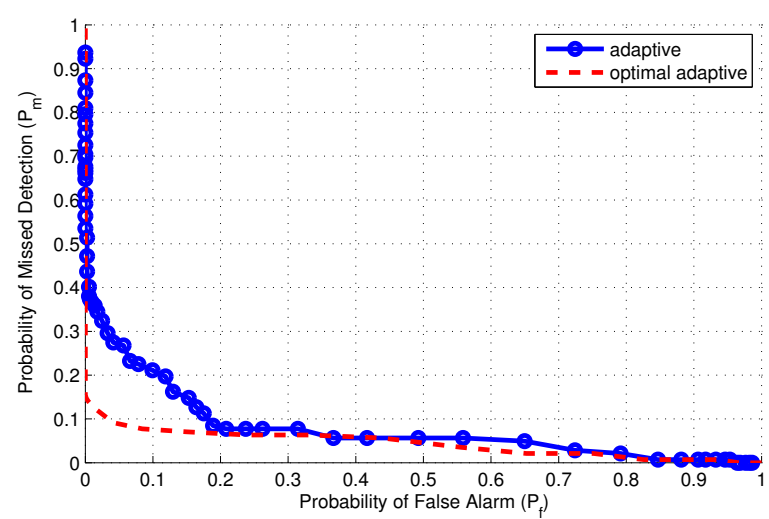

Fig. 6. Adaptive Threshold vs. Optimum Threshold

adaptive threshold which is dependent of minimum number of parameters. Critical parameters of the adaptive threshold is introduced and the detection performance is investigated. Assumption of uniform SNR through the detection band is the main limitation within the proposed study, which is also the subject of further research in the area of adaptive threshold estimation.

\section{Acknowledgment}

This study is supported by Qatar Telecom (Qtel) and Qatar National Research Fund (QNRF).

\section{REFERENCES}

[1] Spectrum Efficiency Working Group, "Report of the Spectrum Efficiency Working Group," Spectrum Policy Task Force, Washington, DC, Tech. Rep., November 2002

[2] "Federal Communications Comission, Tech. Rep.." In Et docket no. 03322. Notice of Proposed Rule Making and Order, Washington, DC , December 2003.

[3] D. Cabric, S. M. Mishra, and R. W. Brodersen, "Implementation issues in spectrum sensing for cognitive radios," In Proc. IEEE Asilomar Conf. on Signals, Syst., Computers, vol. 1, pp. 772-776, Pacific Grove, CA, U.S.A., Nov. 2004.

[4] A. Sahai, N. Hoven, R. Tandra, "A Closed Solution for an Integrated Broadband Mobile System (IBMS)", In Proc. of Intl. Conf. on Universal Personal Communications (ICUPC'96) pp. 707-711 Proc. of Allerton Conference, Monticello, Oct 2004.

[5] D. Cabric, A. Tkachenko, and R. W. Brodersen, "Spectrum Sensing Measurements of Pilot, Energy, and Collaborative Detection," In Proc. IEEE Military Communications Conference pp. 1-7, Washington D. C., U.S.A., 23-25 Oct. 2006.

[6] Arslan, H. and Gorcin, A., "Cognitive Radio and Software Defined Radio: Signal Processing Perspectives," In IEEE 16th Signal Processing, Communication and Applications Conference, 2008, SIU 2008, Didyma, Aydin, Turkey, 20-22 April, 2008.

[7] S. M. Mishra, A. Sahai, and R. W. Broderson, "Cooperative sensing among Cognitive Radios," In Proc. of the IEEE International Conference on Communications, ICC 2006, Istanbul, Turkey, June 11-15 2006.

[8] Z. Quan, S. Cui, A. H. Sayed and H. V. Poor, "Optimal Multiband Joint Detection for Spectrum Sensing in Cognitive Radio Networks," In IEEE Transactions on Communications vol. 57, number 3, pp. 1128-1140, 2009

[9] J. Vartiainen, J. J. Lethomäki and H. Saarnisaari, "Double-Threshold Based Narrowband Signal Extraction," In IEEE 61st Vehicular Technology Conference, VTC 2005-Spring vol. 2, Stockholm, Sweden, May 30 - June 1,2005

[10] J. Zhu, Z. Xu, F. Wang, B. Huang and B. Zhang, "Double Threshold Energy Detection of Cooperative Spectrum Sensing in Cognitive Radio," In IEEE 3rd International Conference on Cognitive Radio Oriented Wireless Networks and Communications,CrownCom 2008 pp. 1-5, Singapore, 15-17 May 2008

[11] W. Han, J. Li, Z. Li, J. Yao and D. Chen, "Double Thresholds for Judgment in Cognitive Spectrum Sensing," In IEEE 19th International Symposium on Personal, Indoor and Mobile Radio Communications, PIMRC 2008, pp. 1-5, Cannes, France, 15-18 September 2008.

[12] D. Miao, F. Zhiyong, C. Huying and Y. Yanjun, "An Adaptive Threshold Method for Data Processing in Spectrum Occupancy Measurements," In IEEE 69th Vehicular Technology Conference, VTC 2009-Fall Anchorage, AL, U.S.A 20-23 September, 2009.

[13] J. W. Lee, J. H. Kim, H. J. Oh and S. H. Hwang, "Energy Detector using Adaptive-Fixed Thresholds in Cognitive Radio Systems," In 14th AsiaPacific Conference on Communications,APCC 2008 Akihabara, Tokyo, Japan, 14-16 October 2008

[14] H. Urkowitz, "Energy detection of unknown deterministic signals," In Proceedings of IEEE vol. 55, pp. 523-531, April 1967.

[15] Z. Ye, G. Memik, J. Grosspietsch, "Energy Detection Using Estimated Noise Variance for Spectrum Sensing in Cognitive Radio Networks," In IEEE Wireless Communications and Networking Conference, WCNC 2008 pp. 711-716, Las Vegas, Nevada, 31 March - 3 April, 2008

[16] M. P. Oliveri, G. Barnett, A. Lackpour, A. Davis, and P. Ngo "A Scalable Dynamic Spectrum Allocation System with Interference Mitigation For Teams Of Spectrally Agile Software Defined Radios," In New Frontiers in Dynamic Spectrum Access Networks, (DySPAN 2005), pp. 170-179, Baltimore, Maryland, U.S.A., Nov. 2005.

[17] M. Sezgin, B. Sankur, "A Survey over Image Thresholding Techniques and Quantitative Performance Evaluation," In J. Electron. Imag., vol.13, no.1, pp. 146-165, Jan. 2004. 